ORIGINAL

\title{
UTILIZAÇÃO DE FRUTOOLIGOSSACARÍDEOS NA ELABORAÇÃO DE PÃO DE FORMA SEM AÇÚCAR
}

\section{USE OF FRUCTOOLIGOSACCHARIDES IN ELABORATION OF SUGAR FREE BREAD}

\author{
Maria T. P. Silva ${ }^{1 *}$, Camila B. Silva ${ }^{2}$, Isis W. Paleo ${ }^{3}$, Yoon K. Chang ${ }^{4}$ \\ Recibido para evaluación: Marzo 8 de 2010 - Aceptado para publicación: Junio 8 de 2010
}

\begin{abstract}
RESUMO
Os frutooligossacarídeos (FOS) são considerados prebióticos e têm 60\% de doçura em relação ao açúcar. O objetivo deste trabalho foi avaliar as características tecnológicas de pães de forma sem açúcar obtidos com o uso de FOS como substituto de açúcar. Foi realizada a composição química e as propriedades reológicas da farinha de trigo. Os pães de forma sem açúcar foram obtidos com três níveis de FOS (F1 = 3,0\%, F2 = $4,5 \%$ e F3 = 6,0\%, em relação ao peso de farinha) e comparados com um pão controle com açúcar (F0). Os parâmetros analisados foram: expansão da massa, volume específico, cor do miolo (luminosidade, croma e tonalidade) e textura do miolo (firmeza, mastigabilidade, elasticidade e coesividade). O melhor pão de forma sem açúcar e com FOS foi selecionado pelo teste de Tukey $(p<0,05)$ e análise de componentes principais (ACP). A farinha de trigo foi considerada com características adequadas para produção de pães. O teste de expansão da massa mostrou que a ausência de açúcar nos pães contendo FOS não prejudicou o processo fermentativo. Os pães sem açúcar e com FOS apresentaram significativamente menor volume específico e mastigabilidade em relação ao F0, o que foi confirmado pela ACP. A ACP também mostrou que F2 apresentou as melhores caracterísiticas em compação a F1 e F3. Como conclusão, este estudo mostrou que é possível produzir pães de forma sem açúcar usando 4,5\% de FOS (F2) como substituto de açúcar e mais benéficos à saude.
\end{abstract}

Palavras chave: Pão de forma sem açúcar, frutooligossacarídeos, prebióticos, características tecnológicas.

\begin{abstract}
Fructoologosaccharides (FOS) are considered prebiotics and have $60 \%$ of sucrose sweetness. The aim of this study was to evaluate technological characteristics of sugar free breads obtained with the use of FOS as a

${ }^{1}$ Professora Adjunto da Faculdade de Nutrição da Universidade Federal de Alfenas-MG -UNIFAL-MG. End: Av Gabriel Monteiro da Silva, 700, Alfenas-MG, Brasil, e-mail*: maria.pedrosa@unifal-mg.edu.br

${ }^{2}$ Universidade Estadual de Campinas/UNICAMP - Campinas, SP - Brasil, Faculdade de Engenharia de Alimentos/FEA e-mail: camila_bsilva@yahoo.com.br

${ }^{3}$ Universidade Estadual de Campinas/UNICAMP - Campinas, SP - Brasil, Faculdade de Engenharia de Alimentos/FEA e-mail: isiswp@gmail.com.

${ }^{4}$ Universidade Estadual de Campinas/UNICAMP - Departamento de Tecnologia de Alimentos/DTA/FEA, Campinas, SP Brasil, e-mail:yokic@fea.unicamp.br
\end{abstract}


sugar substitute. The chemical composition and rheological properties of the wheat flour were determined. The sugar free breads were obtained with three levels of $\mathrm{FOS}(\mathrm{F} 1=3.0 \%, \mathrm{~F} 2=4.5 \%$ and $\mathrm{F} 3=6.0 \%$ in wheat flour basis) and compared with control bread with sugar (F0). The analyzed parameters were: mass expansion, specific volume, crumb color (luminosity, chromo and hue) and texture (firmness, chewiness, springiness and cohesiveness). The best free sugar bread with FOS was selected by using Tukey's test $(p<0.05)$ and the principal component analysis (PCA). The wheat flour was considered within the expected values for use in bread production. The mass expansion test showed that the absence of sugar in breads containing FOS did not inhibit the fermentative process. Sugar free breads with FOS had significantly lower specific volume and chewiness compared to F0, which was confirmed by the PCA. PCA also showed that F2 have best characteristics compared to F1 and F3. As conclusion this study presented the possibility to produce sugar free breads using $4.5 \%$ of FOS as a sugar substitute and to make breads more beneficial to health.

Key-words: Sugar free bread, fructooligosaccharides, prebiotics, technological characteristics.

\section{INTRODUÇÃO}

O pão é uma fonte importante de carboidratos, proteínas e vitaminas (Pomeranz 1987), porém apresenta alto índice glicêmico e baixo conteúdo em fibras (Goñi et al. 1997, Menezes et al. 2009). O consumo de pães preparados com grão inteiro, multigrãos e com fibras tende a aumentar (Faridi e Faubion 1995), uma vez que as pessoas estão buscando alimentos mais benéficos à saúde com redução do consumo de carboidratos simples, gorduras e colesterol, para evitar doenças como obesidade e diabetes mellitus (Ebbeling et al. 2003).

Em produtos de panificação, o uso de grão inteiro e/ou fibras, a substituição do açúcar e da gordura, pode levar a diluição das proteínas formadoras de glúten, afetando a qualidade da massa, como absorção de água e tempo de fermentação, já no produto final pode levar a diminuição do volume específico, aumento da firmeza do miolo, aparência mais escura e textura modificada (Wang et al. 2002; Gomez et al. 2003, Sangnark e Noomhorm 2004).

A busca por fontes de fibras que mantêm a qualidade final do pão tem mostrado que os frutooligossacarídeos (FOS) apresentam as vantagens de alta solubilidade e baixo peso molecular, podendo ser adicionado ao produto sem mudanças nas condições de processo (Passos e Park 2003).

Os frutooligossacarídeos são conhecidos como fibras prebióticas, uma vez que adicionados aos alimentos promovem o crescimento de bactérias benéficas ao sistema digestório do hospedeiro (Lamsal e Faubion 2009). Os produtos à base de cereais têm sido extensivamente pesquisados para a inserção de prebióticos, pois apresentam longa estabilidade durante o armazenamento, avaliação sensorial que tem sido benéfica para estes produtos e grande consumo mundial (Charalampopoulos et al. 2002; Lamsal e Faubion 2009). A dose diária estabelecida para consumo de FOS no Japão é de $0,8 \mathrm{~g} \mathrm{Kg}^{-1}$ de peso corpóreo e em muitos países possuem o status GRAS (Generally Recognized as Safe), porém a ingestão de 20-30 g por dia pode desencadear desconforto abdominal, como flatulência (Bouhnik et al. 1999).

Devido às suas propriedades gelificantes e espessantes, tanto a inulina quanto o FOS são 
muito úteis como ingredientes alimentares e tiveram seus usos difundidos nos últimos anos em alimentos como pães, queijos processados e lacticínios (Williams 1999; Passos e Park 2003). Esses compostos têm excelentes propriedades tecnológicas, sabor e textura e podem ser simplesmente adicionados ou usados para substituir outros carboidratos (Freitas 2000). O objetivo deste trabalho foi avaliar as características tecnológicas de pães de forma sem açúcar obtidos com o uso de FOS nas concentrações de 3, 4,5 e 6\% como substituto de áçucar.

\section{MATERIAL E MÉTODOS}

\section{Ingredientes}

Foram utilizadas como matérias-primas: farinha de trigo enriquecida com ferro e ácido fólico, fornecida pela Cargill, Tatuí-SPBrasil; xarope de frutooligossacarídeos (FOS), BIOLIGO $^{\circledR} 1101$, contendo $80 \%$ de FOS e pH = 5,0, fornecido pela Corn Products do Brasil; fermento biológico instantâneo, fornecido pela AB Brasil ${ }^{\circledR}$, Jundiaí-SP-Brasil; e foram adquiridos gordura vegetal (marca Saúde ${ }^{\circledR}$ ), açúcar refinado (marca União ${ }^{\circledR}$ ) e sal (marca Cisne $^{\circledR}$ ) no comércio de Campinas-SP, Brasil.

\section{Caracterização da farinha de trigo Composição centesimal}

A determinação dos teores de umidade, proteína, cinzas e fibra bruta foi realizada segundo os métodos 44-15A, 46-12, 08-01 e 32-10 da AACC (1995), respectivamente. A quantificação lipídica foi obtida de acordo com o método do IAL (2005) e carboidratos determinado por diferença, segundo a equação:

Carboidratos totais $(\%)=[100$ - (umidade + lipídeos + proteína + cinzas].

\section{Características reológicas}

As análises reológicas da farinha de trigo foram:

- Farinografia (Método 54-21, AACC 1995), foi utilizado farinógrafo Brabender e avaliados os parâmetros absorção de água $(\%)$, tempo de chegada (min), tempo de desenvolvimento (min) e estabilidade ( $\mathrm{min}$ ) da massa.

- Extensigrafia (Método 54-10, AACC 1995) foi utilizado extensógrafo Brabender, que é a medida e o registro da resistência da massa à extensão, enquanto ela é esticada a uma velocidade constante, com análise dos parâmetros: extensibilidade, resistência à extensão e resistência máxima.

- Índice de glúten e teores de glúten seco e úmido, de acordo com o método n 38-12 (AACC 2000), no sistema Glutomatic.

\section{Preparo do pão de forma controle e dos pães de forma sem açúcar e com fos}

Os pães de forma foram produzidos segundo metodologia descrita por El-Dash (1978). Na tabela 1 estão descritas as formulações, onde constam os pães controle, contendo açúcar na formulação (FO) e pães sem açúcar e com FOS concentrações de 3,0 (F1); 4,5 (F2) e 6,0\% (F3).

Os pães de forma foram produzidos na planta piloto de panificação do Departamento de Tecnologia de Alimentos, da Faculdade de Engenharia de Alimentos, da Universidade Estadual de Campinas-SP, Brasil. Foi usado o método de massa direta modificado, que consistiu da mistura dos ingredientes utilizados na formulação em masseira (Hyppolito automática espiral HAE10) com haste tipo gancho. Esta etapa foi realizada em duas fases: velocidade lenta (90 rpm) por 9 minutos e velocidade rápida (180 rpm) por 7 minutos, 
Silva - Pães de forma com frutooligossacarídeos

Tabela 1. Formulações dos pães de forma produzidos com frutooligossacarídeos (FOS)

\begin{tabular}{lcccc}
\hline \multirow{2}{*}{$\begin{array}{l}\text { Ingrediente } \\
\text { Formulação }\end{array}$} & $\begin{array}{c}\text { Controle } \\
\text { F0 (\%) }\end{array}$ & \multicolumn{3}{c}{ Frutooligossacarídeo } \\
\cline { 3 - 5 } & & F1(\%) & F2(\%) & F3(\%) \\
\hline Farinha de trigo & 100 & 100 & 100 & 100 \\
Água * & 68 & 68 & 68 & 68 \\
Sal * & 2 & 2 & 2 & 2 \\
Açúcar * & 5 & 0 & 0 & 0 \\
FOS * & 0 & 3,0 & 4,5 & 6,0 \\
Fermento * & 3 & 3 & 3 & 3 \\
Gordura Vegetal * & 3 & 3 & 3 & 3 \\
\hline
\end{tabular}

*\% em relação ao peso de farinha de trigo em base úmida

até completo desenvolvimento do glúten. Após a mistura, a massa foi deixada descansar por 10 minutos, dividida em porções de 400 g. A modeladora (Hyppolito) auxiliou na modelagem dos pães, que foram colocadas em formas de folha galvanizada de ferro $(22 \times 11$ cm) e levados para câmara de fermentação (Maquip) com controle de temperatura (29-31 oC) e umidade relativa (85 - 90\%) por 100min. Após a fermentação, realizou-se o assamento em forno (Hyppolito), por 20 minutos a 180 oC. Os pães foram desenformados e mantidos à temperatura ambiente por aproximadamente 2 horas. Em seguida, acondicionados em embalagens plásticas e mantidos em temperatura ambiente até a realização das análises.

\section{Avaliação da qualidade dos pães de forma Expansão da massa}

Foi realizada, em triplicata, onde a massa preparada para fazer o pão foi dividida em amostras de $50 \pm 0,01 \mathrm{~g}$ para teste de expansão de massa. As amostras foram moldadas para serem colocadas em uma proveta de $250 \mathrm{~mL}$. As provetas foram deixadas à temperatura ambiente e o volume foi medido nos tempos 0, 45, 90, $135 \mathrm{~min}$, segundo método descrito por Sangnark e Noomhorm (2004).

\section{Volume específico do pão}

A massa dos pães foi determinada, em triplicata, em balança de precisão e expressa em gramas, sendo o volume total obtido pelo método de deslocamento de sementes de painço e o volume específico pela divisão do volume do pão $(\mathrm{cm} 3)$ pela sua massa $(\mathrm{g})$, segundo método 72-10 da AACC (2000).

\section{Coloração do miolo do pão}

Cada pão foi cortado em fatias de 2,5 cm de espessura. Foram utilizados 5 pães de cada formulação e quatro fatias foram retiradas do centro de cada amostra para a avaliação da coloração do miolo.

A quantificação de cor foi feita utilizando-se um espectrofotômetro Color Quest II Hunterlab (Reston, VA, EUA), segundo o sistema de cor CIE-Lab. Determinando-se os valores $L^{*}$ ou luminosidade [(zero (escuro) / 100 (claro)], a* (verde -/ vermelho +) e b* (azul- /amarelo+), 
segundo GIESE (2000), sendo que os valores de a* e b* foram usados para obter o croma $\left(C^{*}\right)$ e o ângulo de tonalidade (hab), de acordo com Minolta (2006) que apresenta as seguintes equações:

$$
\begin{aligned}
& C^{*}=\sqrt{\left(a^{*}\right)^{2}+\left(b^{*}\right)^{2}} \\
& \text { hab }=\tan ^{-1}\left\{b^{*} / a^{*}\right\}
\end{aligned}
$$

\section{Textura do pão}

Foi realizada a Análise do Perfil Textura (TPA), no analisador de textura TA-XT2i, onde foi utilizado o software XTRA Dimension, marca Stable Micro System (Haslemere, Surrey, Inglaterra) (Stable Micro Systems 1997). Para obtenção dos resultados foram utilizados três pães de cada tratamento e 10 fatias centrais de cada pão, totalizando 15 repetições. Foram colocadas duas fatias do miolo do pão no texturômetro, com altura de $2,5 \mathrm{~cm}$ cada. O texturômetro foi ajustado, segundo técnica descrita pelo fabricante, nas seguintes especificações: compressão: $5 \mathrm{~mm}$, sonda (probe): diâmetro 25,4 mm, força "threshold" $=20 \mathrm{~g}$, distância "threshold" $=0,50 \mathrm{~mm}$, força de contato $=5,0 \mathrm{~g}$, velocidade de teste $=2,0$ $\mathrm{mm} \mathrm{s}^{-1}$, velocidade do pré e pós teste $=5,0 \mathrm{~mm}$ $\mathrm{s}^{-1}$, distância $=5,0 \mathrm{~mm}$, ciclo até contagem de 10 e com medida de força em compressão. Os parâmetros analisados foram firmeza $(\mathrm{N})$, elasticidade, coesividade e mastigabilidade.

\section{Análise estatística}

As análises físico-químicas e reológicas foram realizadas em triplicata, já a análise de cor foi realizada em 5 pães de cada formulação e a textura foi feita com 3 pães de cada formulação com 5 repetições para cada pão, num total de 15 repetições por tratamento. Os resultados estão apresentados em valores médios e desvio padrão. Para determinar as diferenças entre os tratamentos foi realizado o teste de Tukey $(p<0,05)$ e para obter a informação global dos efeitos do FOS nas características do pão foi realizada a Análise de Componentes Principais (ACP), pelo SAS (2002) e a representação gráfica no Origin 8.1 (2007).

\section{RESULTADOS E DISCUSSÃO}

\section{Composição centesimal da farinha de trigo}

A composição centesimal (\%), em valores médios \pm desvio padrão, da farinha de trigo foi: $12,81 \pm 0,03$ de umidade; 11,50 $\pm 0,01$ de proteína; 0,42 \pm 0,01 de cinzas; 1,34 \pm 0,02 de lipídeos e 73 de carboidratos. Dados concordantes com os encontrados por Schiller (1984), Kulp e Ponte (2000) e Wang et al. (2002). O teor de umidade, cinzas e proteínas classifica a farinha como sendo do Tipo 1 , de acordo com a Instrução Normativa $n^{\circ} 8$, de 02/06/2005 (Brasil 2005).

\section{Características reológicas da farinha de trigo}

A tabela 2 apresenta os resultados das características reológicas da farinha de trigo. A farinha de trigo usada neste trabalho é própria para panificação uma vez que apresenta os parâmetros de farinografia e índice de glúten característicos de uma farinha forte, como os resultados estabelecidos por Pyler (1988), Pizzinatto (1999), Faroni et al. (2002) e Cauvain e Young (2009), já extensografia foi característica de uma farinha média a fraca, sendo que nestes casos, somente o teste de panificação poderá prever a qualidade do pão adequada para produção de pães (El-Dash 1978). 
Tabela 2. Caracterização tecnológica da farinha de trigo

\begin{tabular}{|c|c|c|c|c|}
\hline Análises & Parâmetros & \multicolumn{3}{|c|}{ Resultados } \\
\hline \multirow[t]{4}{*}{ Farinografia } & Absorção (\%) & \multicolumn{3}{|c|}{63,3} \\
\hline & Tempo de chegada (min) & \multicolumn{3}{|c|}{1,8} \\
\hline & Tempo de desenvolvimento (min) & \multicolumn{3}{|c|}{2,8} \\
\hline & Estabilidade (min) & \multicolumn{3}{|c|}{10,0} \\
\hline \multirow[t]{5}{*}{ Extensigrafia } & & $45 \mathrm{~min}$ & 90 min & $135 \mathrm{~min}$ \\
\hline & E (extensibilidade) (mm) & 180 & 185 & 195 \\
\hline & R (resistência à extensão) (UB*) & 170 & 160 & 180 \\
\hline & Rmax (resistência máxima) (UB*) & 290 & 290 & 280 \\
\hline & $\mathrm{D}(=\mathrm{R} / \mathrm{E})$ & 0,94 & 0,86 & 0,92 \\
\hline \multirow[t]{3}{*}{ Glúten } & Úmido (\%) & \multicolumn{3}{|c|}{26,9} \\
\hline & $\operatorname{Seco}(\%)$ & \multicolumn{3}{|c|}{9,0} \\
\hline & Índice & \multicolumn{3}{|c|}{100} \\
\hline
\end{tabular}

*UB = Unidade Brabender

Avaliação da qualidade dos pães de forma Análise da expansão da massa

Os resultados da análise da expansão da massa estão apresentados na tabela 3. Verifica-se que no tempo zero, as formulações contendo FOS apresentaram significativamente menor volume, indicando baixa produção de gás inicial, mas ao final do tempo de fermentação, elas apresentaram maior volume em comparação com a massa controle (F0).

Este maior volume de massa apresentado pelos pães com FOS durante a fermentação pode ser devido à ação das enzimas amilases e invertases que podem hidrolisar o amido danificado e o FOS, respectivamente, e em ambos os casos pode haver liberação de glicose, que será usado pelas leveduras.

O amido danificado é capaz de absorver água e ser hidrolisado pelas enzimas amilolíticas presentes na farinha de trigo e, consequentemente na massa do pão, que afeta o volume e a cor da crosta do produto final (Farrand 1964; Osborne e Douglas 1981 e Barrera et al. 2007).

A hidrólise do FOS pela ação da invertase, enzima presente no fermento, que aumenta a disponibilidade de açúcares para fermentação, foi constatada em vários trabalhos, tanto que linhagens de leveduras que não produzem invertase para uso em panificação têm sido desenvolvidas para pães contendo FOS ou inulina (Nakatomi et al. 1987; Oda e Ouchi 1991).

\section{Volume específico dos pães de forma}

O volume específico dos pães de forma pode ser visualizado na tabela 4. Como pode ser observado, não houve diferença significativa $(p<0,05)$ entre os volumes específicos dos 
Tabela 3. Avaliação da expansão em volume $(\mathrm{mL})$ de $50 \mathrm{~g}$ da massa dos pães controle e dos pães sem açúcar e com frutooligossacarídeos (FOS)*

\section{Formulações}

Tempo (minutos)**

$$
0 \quad 45 \quad 90
$$

135

\begin{tabular}{lllll}
\hline F0 (Controle) & $57^{\mathrm{a}} \pm 0,78$ & $112^{\mathrm{c}} \pm 0,97$ & $130^{\mathrm{b}} \pm 1,51$ & $140^{\mathrm{c}} \pm 1,98$ \\
F1 $(3 \%$ de FOS $)$ & $51^{\mathrm{b}} \pm 1,35$ & $120^{\mathrm{a}} \pm 1,52$ & $138^{\mathrm{a}} \pm 1,14$ & $146^{\mathrm{ab}} \pm 1,78$ \\
F2 $(4,5 \%$ de FOS $)$ & $52^{\mathrm{b}} \pm 0,98$ & $117^{\mathrm{a}} \pm 1,02$ & $137^{\mathrm{a}} \pm 1,52$ & $144^{\mathrm{bc}} \pm 1,64$ \\
F3 $(6 \%$ de FOS $)$ & $52^{\mathrm{b}} \pm 1,55$ & $116^{\mathrm{ab}} \pm 1,47$ & $140^{\mathrm{a}} \pm 1,31$ & $150^{\mathrm{a}} \pm 1,78$ \\
\hline
\end{tabular}

* Resultados apresentados em valores médios+desvio padrão

**Médias seguidas de letras diferentes, na vertical, diferem significativamente entre si pelo teste de Tukey $(p<0,05)$

pães F1, F2 e F3 $\left(4,87\right.$ a 4,74 $\left.\mathrm{mL} \mathrm{g}^{-1}\right)$, porém eles foram significativamente menores que F0 $\left(5,19 \mathrm{~mL} \mathrm{~g}^{-1}\right)$ e todos apresentaram valores abaixo do valor máximo esperado para esta característica que é de $6 \mathrm{~mL} \mathrm{~g}^{-1}$, segundo ElDash (1978), pode ser devido as características extensográficas da farinha, que se mostrou com força média a fraca.

Comparando os resultados de expansão da massa (Tabela 3) com o volume específico (Tabela 4) pode-se verificar que apesar da massa com FOS apresentar maiores valores de expansão durante fermentação, esta expansão não se manteve durante o assamento e resultou em pães com menores volumes.

A redução de volume nos pães contendo FOS já era esperada, pois Wang et al. (2002) e Gomez et al. (2003) estudaram o efeito da adição de diferentes fibras na qualidade do pão e verificaram uma redução no volume específico do pão com a adição de fibras, segundo estes autores a interação entre fibras e glúten pode impedir a expansão da massa, devido ao escape do gás durante a fementação e assamento, uma vez que, segundo Collar et al. (2007) e Angioloni e Collar (2009), a fibra dilui o glúten e provoca uma interrupção da matriz amido-glúten prejudicando a qualidade do pão.

\section{Cor instrumental do miolo dos pães de forma}

A cor do miolo dos pães de forma está apresentada na Tabela 4. Em relação ao parâmetro Luminosidade (L) os pães apresentaram a mesma intensidade, com coloração mais perto de 100, indicando que os miolos estavam claros.

Não houve diferenças significativas entre os pães das formulações contendo FOS e o F0 em relação ao croma $\left(\mathrm{C}^{*}\right)$. O croma mostra o grau de saturação da cor, sendo que quanto maior o valor, mais saturada é a cor do produto (Minolta 2006), logo todos os pães apresentaram a mesma intensidade de cor, em relação ao grau de saturação.

Em relação à tonalidade dos pães houve diminuição significativa quando se usou 6\% de FOS (F3) em comparação aos demais pães, indicando que houve diminuição da cor amarela (ângulo 90 graus = amarelo) com inclinação para a vermelha (ângulo $0=$ vermelho), uma vez que o F0 apresentou hab $=85$ e o F3 teve hab=83. 


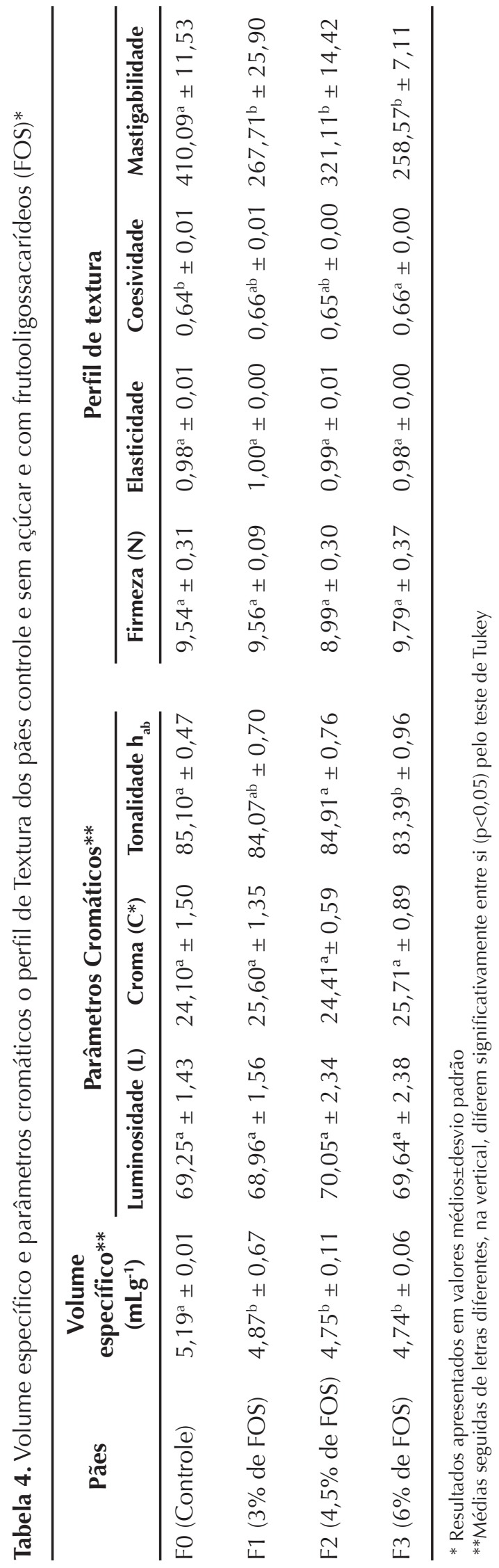


A cor do miolo dos pães de forma constitui um fator muito importante para a sua comercialização, sendo diretamente influenciada pelas matérias-primas que compôem sua formulação e pelas condições de forneamento (Silva 2009). Os resultados apresentados neste estudo estão próximos aos encontrados por Silva (2009) que avaliou a cor de miolo em pães contendo farinha de soja fermentada e encontrou os valores de $L^{*}$ variando de 67 a $72 ; C^{*}$ de 18 a 26 e hab de 81 a 89 , já o pão controle apresentou $L^{*}$ de $70, C^{*}$ de 18 e hab de 89.

\section{Textura}

A tabela 4 apresenta o perfil de textura dos pães. As características de firmeza e elasticidade não apresentaram diferenças significativas entre $\mathrm{O}$ pão FO e os contendo FOS. A coesividade foi semelhante entre os pães contendo FOS, porém houve diferença significativa entre o F0 e o F3. O maior efeito da adição de FOS foi observado na característica de mastigabilidade, sendo que o F0 apresentou significativamente o maior valor em relação aos demais pães com FOS.

Pode-se verificar ainda na tabela 4 que o aumento da concentração de FOS, de 3 a 6\%, não afetou o perfil de textura dos pães sem açúcar, pois eles não apresentaram diferenças significativas para as características analisadas.

A firmeza é o principal parâmetro de avaliação de um pão, logo os resultados mostraram que mesmo com redução do volume dos pães com FOS, não houve aumento da firmeza, sendo que Peressini e Sensidoni (2009) encontraram resultados diferentes para pães com inulina, que apresentaram diminuição de volume e aumento da firmeza com o aumento da concentração de 5 a 7\% de inulina no pão.
Lima et al. (2002) relataram um aumento significativo na firmeza de pães de forma, cortados em cubos, preparados com adição de farelo de arroz em substituição parcial à farinha de trigo, em concentrações de 10 e $20 \%$, em relação a um controle sem farelo. O aumento de firmeza em pães de forma também foi relatado por Silva (2009) com o uso de farinha de soja fermentada.

Portanto o uso de FOS em pães de forma, em concentrações de 3 a $6 \%$ apresenta resultados promissores de textura, em relação a firmeza, mantendo a mesma qualidade em relação ao pão controle, se for comparado as resultados obtidos para outros tipos de fibras e ingredientes (Lima et al. 2002, Silva 2009, Peressini e Sensidoni 2009).

\section{Análise de componente principais (ACP)}

Como as avaliações de qualidade tecnológicas feitas pelo teste de Tukey $(p<0,05)$ entre os pães controle e os contendo FOS só mostraram diferenças no volume específico, mastigabilidade e luminosidade, mas não conseguiram apontar a melhor concentração de FOS para uso nos pães, foi realizada a ACP, que pode ser considerada como um esforço para descobrir dependências aproximadamente lineares entre as variáveis (SAS 2002).

Pode-se observar, na figura 1, que $58 \%$ da variação dos resultados foi explicado pelo CP1 e 25\% pelo CP2, resultando um total de $83 \%$ de explicação da variação dos dados por estes dois componentes, logo a ACP pode ser usada para explicar os resultados obtidos neste trabalho.

No CP1, a maior variabilidade entre as 


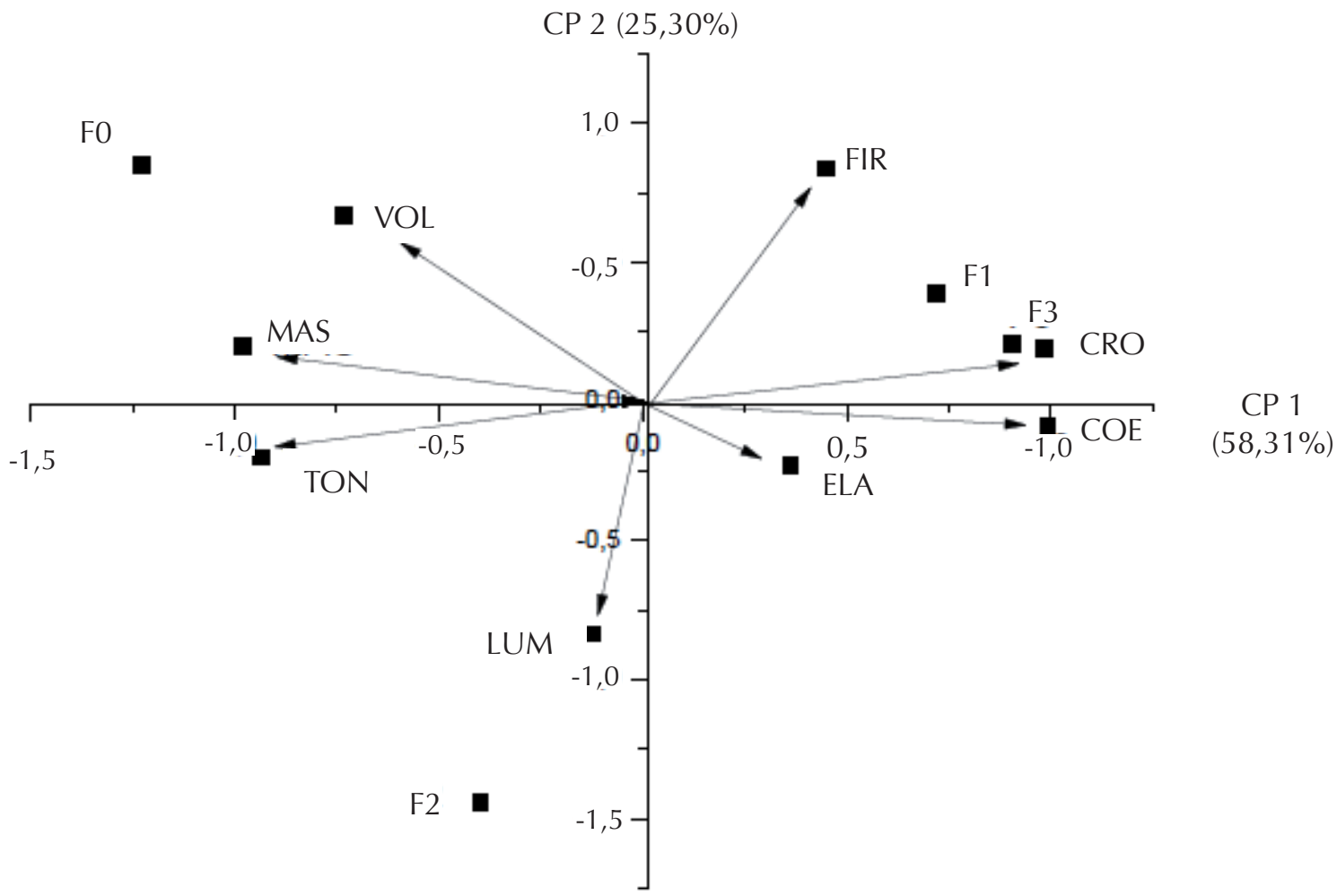

Figura 1. Análise de Componentes Principais (ACP) das características tecnológicas do pão controle (F0) e dos pães sem açúcar e com FOS ( $F 1=3 \%, F 2=4,5 \%$ e $F 3=6 \%$ )

CP: componente principal, VOL: volume específico, MAS: mastigabilidade, FIR: firmeza, ELA: elasticidade, COE: coesividade, LUM: luminosidade, CRO: croma e TON: tonalidade.

amostras foi devido às características dos vetores relacionados ao croma (CRO), a coesividade (COE), a tonalidade (TON), a mastigabilidade (MAS) e a elasticidade (ELA). No entanto, o volume (VOL) e firmeza (FIR) estão influenciando tanto o CP1 quanto o CP2.

Quando se analisa os pães de forma deve-se observar que quando eles estão próximos entre si, significa que são similares em relação aos atributos julgados, já quando distantes uns dos outros, apresentam altas diferenças entre eles. Portanto, o pão controle F0 se apresenta distante e oposto ao F1 e F3, em relação ao CP1. Já o F2 se encontra distante de todos os pães, porém mais próximo de CP2 e das características explicadas por ele. A localização de cada processo sugere também, qual atributo se apresenta em maior intensidade nele.

Os pães F0, F1 e F3 estão mais perto do CP1 e influenciando os atributos diretamente relacionados a ele, como os vetores de VOL, MAS eTON que estão próximos ao pão F0, esta formulação influenciou os maiores valores, no entanto os menores valores foram devido aos pães F1 e F3, sendo que estes pães também afetaram os maiores valores de FIR, CRO, COE e ELA.

A LUM foi à característica mais afetada pelo pão F2, que não teve muita influência nos demais parâmetros de qualidade, uma vez que está mais relacionado ao CP2 e se encontra 
afastado dos demais vetores. Esta análise indica que a produção de pão de forma sem açúcar e com 4,5\% de FOS poderá produzir pães que terão pouco efeito negativo nos atributos de qualidade, quando comparados com um pão de forma contendo açúcar.

\section{CONCLUSÕES}

Este trabalho mostrou ser possível produzir pães sem açúcar e com adição de 4,5\% de FOS com poucas variações nas características de qualidade em relação a um pão de forma obtido com açúcar em sua formulação, o que aumenta as possibilidades de uso de fibras solúveis prebióticas em produtos de panificação, para torná-los mais benéficos à saúde.

\section{AGRADECIMENTOS}

Ao Programa Institucional de Bolsas de Iniciação Científica/Conselho Nacional de Desenvolvimento Científico e Tecnológico (Pibic/CNPq) e às empresas Corn Products Brasil e Cargill Brasil.

\section{REFERÊNCIAS}

\section{AACC - American Association of Cereal} Chemists. 1995. Approved methods of American Association of Cereal Chemists. St. Paul, 9a ed. 2v.

\section{AACC. American Assosciation of Cereal} Chemists. 2000. Approved methods of the AACC. St. Paul, 10 ed. 2v.

Angioloni, A. e Collar, C. 2009. Gel, dough and fibre enriched fresh breads: Relationships between quality features and staling kinetics. Journal of Food Engineering 91(4): 526-532.

Barrera, G., Pérez, G., Ribotta, P. e León, A. 2007. Influence of damaged starch on cookie and bread-making quality. European Food Research and Technology 225(1):1-7.

Bouhnik, Y., Vahedi, K., Achour, L., Attar, A., Salfati, J., Pochart, P., Marteau, P., Flourie, B., Bornet, F. e Rambaud, J. 1999. Short-chain fructooligosaccharide administration dose-dependently increases fecal bifidobacteria in healthy humans. Journal of Nutrition 129(1):113-6.

Brasil. 2005. Ministério da Agricultura e do Abastecimento. Instrução Normativa no 8, de 02/06/2005. Aprovar o regulamento técnico de identidade e qualidade da farinha de trigo. http:// www.anvisa.gov.br [25 maio 2010].

Cauvain, S. e Young, L. 2009. Tecnologia de panificação. Barueri, ed. Manole. 440p.

Charalampopoulos, D., Pandiella, S. e Webb, C. 2002. Growth studies of potentially probiotic lactic acid bacteria in cereal-based substrates. Journal of Applied Microbiology 92(5):851-859.

Collar, C., Santos, E. e Rosell, M., 2007. Assessment of rheological profile of fibre enriched bread doughs by response surface methodology. Journal of Food Engineering 78(3):820-826. 
Ebbeling, C., Leidig, M., Sinclair, K., Hangen, J. e Ludwig, D. 2003. A ReducedGlycemic Load Diet in the Treatment of Adolescent Obesity. Archives of Pediatrics \& Adolescent Medicine 157(8):773-779.

El-Dash, A. 1978. Standardized mixing and fermentation procedure for experimental baking test. Cereal Chemistry 55(4):436-446.

Faridi, H. e Faubion, J. 1995. Wheat usage in North America. Em: Faridi, H., Faubion, J.M., (Ed), Wheat end uses around the world. American Association of Cereal Chemists, St Paul, p1-41.

Faroni, L., Berbert, P., Martinazzo, A. e Coelho, E. 2002. Qualidade da farinha obtida de grãos de trigo fumigados com dióxido de carbono e fosfina. Revista Brasileira de Engenharia Agrícola e Ambiental 6(2): 354-357.

Farrand, E. 1964. Flour properties in relation to the modern bread process in the United Kingdom, with special reference to a-amylaseand starch damage. Cereal Chemistry, 41(2):98111.

Freitas, D. 2000. Efeito da adição de pectina e frutooligossacarídeo como ingredientes funcionais no suco misto de cenoura e laranja. Tese de Mestrado. Universidade Estadual de Campinas, Campinas.
Giese, J. 2000. Color measurement in foods as a quality parameter. Food Technology 54(2):62-63.

Gomez, M., Ronda, F., Blanco, C., Caballero, P. e Apesteguia, A. 2003. Effect of dietary fibre on dough rheology and bread quality. European Food Research and Technology 216(1):5156.

Goñi, I., Garcia-Alonso, A. e Saura-Calixto, F. 1997. A starch hydrolysis procedure to estimate glycemic index. Nutrition Research 17(3):427-437.

IAL - Instituto Adolfo Lutz. 2005. Normas Analíticas do Instituto Adolfo Lutz: Métodos Químicos e Físicos para Alimentos e Bebidas, Ed. São Paulo, São Paulo, 533p.

Kulp, K. e Ponte, J. 2000. Handbook of Cereal Science and Technology. Ed. Marcel Dekker, 2 ed, New York, 790p.

Lamsal, B. e Faubion, J. 2009. The beneficial use of cereal and cereal components in probiotic foods. Food Reviews International 25(2):103-114.

Lima, I., Guraya, H. e Champagne, E. 2002. The functional effectiveness of reprocessed rice bran as an ingredient in bakery products. Nahrung / Food 46(2):112 -117. 
Menezes, E., Giuntini, E., Dan, M. e Lajolo, F. 2009. New information on carbohydrates in the Brazilian Food Composition Database. Journal of Food Composition and Analysis 22(5):446-452.

Minolta. 2006. Minolta CO. LTD. The essentials of imaging, Manual Guide. Japan. p18-21.

Nakatomi, Y., Hara, K., Umeda, F., Kono, T. e Niimoto, H. 1987. Novel baker's yeast and process for making bread. United States Patent 4693898. Application Number: 06/848854; Publication Date: $\quad 09 / 15 / 1987, \quad$ http://www. freepatentsonline.com/4693898.html [30 de agosto de 2010].

Oda, Y. e Ouchi, K. 1991. Construction of a sucrose-fermenting bakers' yeast incapable of hydrolysing fructooligosaccharides. Enzyme and Microbial Technolology. 13(6):495-8.

Origin 8.1. 2007. Northampton, MA 01060, U.S.A. www.OriginLab.com [30 de agosto de 2010].

Osborne, B. e Douglas, S. 1981. Measurement of the degree of starch damage in flour by near infrared reflectance analysis. Journal of the Science of Food and Agriculture 32(4):328-332.

Passos, L. e Park, Y. 2003. Frutooligossacarídeos: implicações na saúde humana e utilização em alimentos. Ciência Rural 33(2):385-390.
Peressini, D. e Sensidoni, A. 2009. Effect of soluble dietary fibre addition on rheological and breadmaking properties of wheat doughs. Journal of Cereal Science 49(2):190-201.

Pizzinatto, A. 1999. Qualidade da farinha de trigo: conceito, fatores determinantes e parâmetros de avaliação e controle. Instituto de Tecnologia de Alimentos, Campinas, $72 \mathrm{p}$.

Pomeranz, Y. 1987. Bread around the world. En: Pomeranz, Y. (ed). Modern Cereal Science and Technology. $\mathrm{VCH}$ Publishers, New York, p. 258-333.

Pyler, E. 1988. Baking - Science e Tecnology. 3.ed. vol II. Sosland Publishing Company, Kansas, 1396p.

Sangnark, A. e Noomhorm, A. 2004. Effect of dietary fiber from sugarcane bagasse and sucrose ester on dough and bread properties. Lebensmittel-Wissenschaft und-Technologie, 37(7):697-704.

SAS. 2002. Sas Institute Inc. Cary- USA, versão 9.

Schiller, G. 1984. Bakery flour specifications. Cereal Foods World, 29(10):647-651.

Silva, L. 2009. Efeito da fermentação utilizando Aspergillus oryzae sobre as características funcionais, tecnológicas e físico-químicas da farinha de soja integral e aplicação em pão de forma funcional. Tese de Doutorado. Universidade Estadual de Campinas, Campinas. 
Stable Micro Systems. (1997). User Manual. Textura Analyser modelo TA-XT2i, Godalming, version 6.10 e 7.10 .

Wang, J., Rosell, C. e Barber, C. 2002. Effect of the addition of different fibres on wheat dough performance and bread quality. Food Chemistry, 79(2):221226.
Williams, C. 1999. Effects of Inulin on Lipid Parameters in Humans. Journal of Nutrition 129(7):1471S-1473S. 